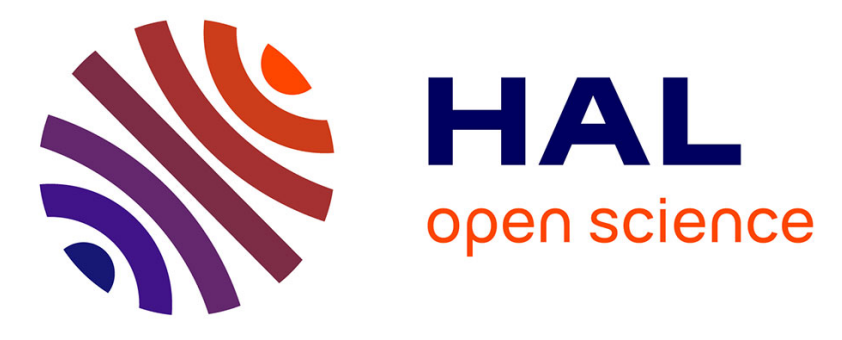

\title{
Walking pattern generators designed for physical collaboration
}

Don Joven Agravante, Alexander Sherikov, Pierre-Brice Wieber, Andrea Cherubini, Abderrahmane Kheddar

\section{- To cite this version:}

Don Joven Agravante, Alexander Sherikov, Pierre-Brice Wieber, Andrea Cherubini, Abderrahmane Kheddar. Walking pattern generators designed for physical collaboration. ICRA: International Conference on Robotics and Automation, May 2016, Stockholm, Sweden. pp.1573-1578, 10.1109/ICRA.2016.7487296 . hal-01274791v2

\section{HAL Id: hal-01274791 \\ https://hal.science/hal-01274791v2}

Submitted on 2 Mar 2016

HAL is a multi-disciplinary open access archive for the deposit and dissemination of scientific research documents, whether they are published or not. The documents may come from teaching and research institutions in France or abroad, or from public or private research centers.
L'archive ouverte pluridisciplinaire HAL, est destinée au dépôt et à la diffusion de documents scientifiques de niveau recherche, publiés ou non, émanant des établissements d'enseignement et de recherche français ou étrangers, des laboratoires publics ou privés. 


\title{
Walking pattern generators designed for physical collaboration
}

\author{
Don Joven Agravante ${ }^{1,4}$, Alexander Sherikov ${ }^{2}$, Pierre-Brice Wieber ${ }^{2}$, Andrea Cherubini ${ }^{1}$, and \\ Abderrahmane Kheddar ${ }^{1,3}$
}

\begin{abstract}
This paper is about the design of humanoid walking pattern generators to be used for physical collaboration. A particular use case is a humanoid robot helping a human to carry large and/or heavy objects. To do this, we construct a reduced model which takes into account physical interaction. This is used in a model predictive control framework to generate separate behaviors for being a follower or a leader. The approach is then validated both on simulation and on the HRP-4 humanoid robot.
\end{abstract}

Index Terms-Humanoid walking, human-humanoid physical interaction, model-predictive control.

\section{INTRODUCTION AND BACKGROUND}

Walking is a large topic of interest within humanoid robotics. This paper is specifically about the generation of dynamically-consistent walking. Perhaps the most prevalent concept in this area is the Zero-Moment Point (ZMP) [1] which encapsulates the essential dynamics of the system. A widely-recognized benchmark is applying preview control on the ZMP, where the future footstep locations are predefined [2]. One of the latest improvements on this method is based on Model Predictive Control (MPC), with constraints on the ZMP, and variable footsteps that are part of the optimization result [3]. Our current work is based on [3], with the foremost contribution that it accounts for and uses physical interaction (i.e., external sustained forces).

Previous works have demonstrated the capability of humanoids to physically collaborate while walking, in particular transporting large or heavy objects [4]-[6]. These were done using the methods described in [2], [3]. In these cases, the walking pattern generator (WPG) does not take into account the physical interaction, which is treated as a disturbance. Since success relies on the robustness of the WPG to this disturbance, impedance control in the arms is commonly added to regulate the interaction forces.

To our knowledge, there have been no works where walking is specifically designed for use in human-humanoid collaboration. The closest to this in literature is taking into account external forces in the WPG model. Several works do this in demonstrations, where a humanoid robot pushes/pulls

\footnotetext{
${ }^{1}$ CNRS-UM LIRMM, Interactive Digital Human group, 161 Rue Ada, 34090 Montpellier, France $\{$ lastname $\}$ @ lirmm. fr.

2INRIA Rhône-Alpes, BIPOP, 655 Avenue de l'Europe - CS 90051, 38334 Montbonnot Cedex, France \{firstnames. lastname\}@inria.fr.

${ }^{3}$ CNRS-AIST, JRL (Joint Robotics Laboratory), UMI 3218/RL, Intelligent Systems Research Institute, AIST Central 1, Umezono 1-1-1, Tsukuba, 305-8560, Japan.

4 has moved to INRIA Rennes - Bretagne Atlantique, Lagadic group, Campus Universitaire de Beaulieu, 35042 Rennes, France.
}

objects while walking [7]-[11]. These present different variations to improve [2], by considering the physical interaction with the object. All rely on simplifying assumptions on the interaction wrench applied on the robot, whereas the full wrench is presented and utilized here. Additionally, all of these works, except for [7], mention the use of impedance/force control on the arms to increase robustness. In [12], the effect of this added impedance control is directly taken into account in the modeling of the MPC, although it is used for postural stability instead of walking. The same concepts were used in [13], for better standing balance during a kinesthetic teaching application. Differently, [13] models the disturbance as a torque on the ZMP. Another closely related work is [14], which builds on [10] in pushing heavy objects. In particular, the range of allowable external forces is obtained from the model considering a pre-defined ZMP, as in [2]. Differently from this, the MPC framework presented here allows to vary both the external forces and ZMP (depending on the objective function weights).

In this work, the full external wrench is taken into account starting from the modeling stage. We then add impedance control on the Center of Mass (CoM), directly in the WPG, in contrast with the two-layer approach of previous works. Finally, a formulation where the external wrench is an output of the WPG (making it a reference to be tracked by the whole-body control) is presented. This may allow the robot to take advantage of the physical interaction to balance itself.

\section{MODELING}

This section and its notation are based on [15]. In comparison, the novelty of this work is to explicitly separate the ground contact forces on the feet $\mathbf{f}_{i}$ and all the other contact forces from the environment, represented as an external wrench in a fixed orientation frame placed on the CoM $\left[\mathbf{f}_{\text {ext }}^{\top} \mathbf{n}_{\text {ext }}^{\top}\right]^{\top} \in \mathbb{R}^{6}$. The external wrench is separated because it will go on to represent physical interaction (with a human or in pushing objects for example). This model is chosen because of its simplicity (as opposed to including the full contact dynamics of the human and/or object). The resulting Newton and Euler equations of motion are now:

$$
\begin{aligned}
m(\ddot{\mathbf{c}}+\mathbf{g}) & =\mathbf{f}_{\mathrm{ext}}+\sum_{i} \mathbf{f}_{i} \\
\dot{\mathbf{L}} & =\mathbf{n}_{\mathrm{ext}}+\sum_{i}\left(\mathbf{p}_{i}-\mathbf{c}\right) \times \mathbf{f}_{i},
\end{aligned}
$$

where $m$ is the mass of the robot, c symbolizes the CoM position in a fixed inertial reference frame, $g$ the acceleration due to gravity, $\mathbf{L}$ the angular momentum and $\mathbf{p}_{i}$ the coplanar 
foot contact points. These equations can be combined to obtain:

$$
\sum_{i} \mathbf{p}_{i} \times \mathbf{f}_{i}=m \mathbf{c} \times(\ddot{\mathbf{c}}+\mathbf{g})+\dot{\mathbf{L}}-\mathbf{n}_{\mathrm{ext}}-\left(\mathbf{c} \times \mathbf{f}_{\mathrm{ext}}\right) .
$$

Dividing (3) by the $z$ component of the feet contact forces while using (1), yields:

$$
\frac{\sum_{i} \mathbf{p}_{i} \times \mathbf{f}_{i}}{\sum_{i} f_{i}^{z}}=\frac{m \mathbf{c} \times(\ddot{\mathbf{c}}+\mathbf{g})+\dot{\mathbf{L}}-\mathbf{n}_{\mathrm{ext}}-\left(\mathbf{c} \times \mathbf{f}_{\mathrm{ext}}\right)}{m\left(\ddot{c}^{z}+g^{z}\right)-f_{\mathrm{ext}}^{z}} .
$$

We assume that the robot is walking on a flat ground, with all contact points between the feet and the ground having the same $z$ coordinate, $p_{i}^{z}=0$, and with a constant height above the ground, so $\ddot{c}^{z}=0$. This allows obtaining a linear relationship between the motion of the CoM and the position of the Center of Pressure. For the sake of simplicity, we also assume here that variations of the angular momentum are negligible, so $\dot{\mathbf{L}}=\mathbf{0}$, and that gravity is orthogonal to the ground, so the constant $\mathbf{g}^{x, y}=\mathbf{0}$. With these assumptions, we can then simplify the $x$ and $y$ components of (4) to get:

$$
\begin{array}{r}
\frac{\sum_{i} f_{i}^{z} \mathbf{p}_{i}^{x, y}}{\sum_{i} f_{i}^{z}}=\left(\frac{m g^{z}}{m g^{z}-f_{\mathrm{ext}}^{z}}\right)\left(\mathbf{c}^{x, y}-\frac{c^{z}}{g^{z}} \ddot{\mathbf{c}}^{x, y}\right) \\
-\mathbf{S}_{\mathrm{mod}}\left(\frac{\mathbf{n}_{\mathrm{ext}}^{x, y}+\left(\mathbf{c} \times \mathbf{f}_{\mathrm{ext}}\right)^{x, y}}{m g^{z}-f_{\mathrm{ext}}^{z}}\right)
\end{array}
$$

where $\mathbf{S}_{\mathrm{mod}}=\left[\begin{array}{cc}0 & -1 \\ 1 & 0\end{array}\right]$

Before moving on with the modeling, it is important to explain the meaning of (5). Firstly, note that the left hand side of the equation is the definition of the Center of Pressure (CoP). Obtaining this was the purpose of the algebraic manipulations so far. Because the ground reaction forces are unilateral (strictly positive), the CoP must belong to the convex hull of the contact points, $\mathbf{p}_{i}$ [15]. Note that the CoP is also known as Zero Moment Point (ZMP) in the literature. Furthermore, the convex hull of the feet contact points is often referred to as the support polygon. The basis of most walking algorithms is to keep the ZMP within the support polygon [2], [3], which enforces consistency with the contact dynamics.

Simplifying further and grouping terms, we end up with the following expression of the ZMP denoted by $\mathbf{z}^{x, y}$ :

$$
\begin{aligned}
\mathbf{z}^{x, y}= & \mathbf{c}^{x, y}-\left(\frac{c^{z}}{g^{z}-\frac{f_{\mathrm{ext}}^{z}}{m}}\right) \ddot{\mathbf{c}}^{x, y} \\
& -\mathbf{S}_{\mathrm{mod}}\left(\frac{\mathbf{n}_{\mathrm{ext}}^{x, y}}{m g^{z}-f_{\mathrm{ext}}^{z}}\right)+\left(\frac{c^{z} \mathbf{f}_{\mathrm{ext}}^{x, y}}{m g^{z}-f_{\mathrm{ext}}^{z}}\right) .
\end{aligned}
$$

When there is no external wrench, this simplifies to:

$$
\mathbf{z}^{x, y}=\mathbf{c}^{x, y}-\left(\frac{c^{z}}{g^{z}}\right) \ddot{\mathbf{c}}^{x, y},
$$

which is the standard expression found in the literature for the ZMP. Some guidelines on how to reduce the impact of an external wrench can be inferred from equation (6): higher robot mass, lower CoM height, external force aligned with the CoM to reduce the corresponding torque.
In order to generate smooth motions of the CoM, we consider that its trajectory is differentiable three times. With $\hat{\mathbf{c}}_{k}=\left[\begin{array}{llllll}c_{k}^{x} & \dot{c}_{k}^{x} & \ddot{c}_{k}^{x} & c_{k}^{y} & \dot{c}_{k}^{y} & \ddot{c}_{k}^{y}\end{array}\right]^{\top}, \mathbf{u}_{k}=\left[\begin{array}{lll}\dddot{c}_{k}^{x} & \dddot{c}_{k}^{y}\end{array}\right]^{\top}, \mathbf{f}_{k}=$ $\left[\begin{array}{llll}n_{k}^{y} & f_{k}^{x} & n_{k}^{x} & f_{k}^{y}\end{array}\right]^{\top}$, we can define the following time-varying discrete time linear model:

$$
\begin{aligned}
\hat{\mathbf{c}}_{k+1} & =\mathbf{A}_{k} \hat{\mathbf{c}}_{k}+\mathbf{B}_{k} \mathbf{u}_{k} \\
\mathbf{z}_{k+1} & =\mathbf{D}_{k+1} \hat{\mathbf{c}}_{k+1}+\mathbf{G}_{k+1} \mathbf{f}_{k+1} \\
& =\mathbf{D}_{k+1} \mathbf{A}_{k} \hat{\mathbf{c}}_{k}+\mathbf{D}_{k+1} \mathbf{B}_{k} \mathbf{u}_{k}+\mathbf{G}_{k+1} \mathbf{f}_{k+1},
\end{aligned}
$$

where

$$
\begin{gathered}
\mathbf{A}_{k}=\left[\begin{array}{llllll}
1 & T & \frac{T^{2}}{2} & 0 & 0 & 0 \\
0 & 1 & T & 0 & 0 & 0 \\
0 & 0 & 1 & 0 & 0 & 0 \\
0 & 0 & 0 & 1 & T & \frac{T^{2}}{2} \\
0 & 0 & 0 & 0 & 1 & T \\
0 & 0 & 0 & 0 & 0 & 1
\end{array}\right], \mathbf{B}_{k}=\left[\begin{array}{ccc}
\frac{T^{3}}{6} & 0 \\
\frac{T^{2}}{2} & 0 \\
T & 0 \\
0 & \frac{T^{3}}{6} \\
0 & \frac{T^{2}}{2} \\
0 & T
\end{array}\right], \\
\mathbf{D}_{k}=\left[\begin{array}{ccccccc}
1 & 0 & -\frac{m c_{k}^{z}}{m g^{z}-f_{k}^{z}} & 0 & 0 & 0 \\
0 & 0 & 0 & 1 & 0 & -\frac{m c_{k}^{z}}{m g^{z}-f_{k}^{z}}
\end{array}\right], \\
\mathbf{G}_{k}= \\
{\left[\begin{array}{ccccc}
\frac{1}{m g^{z}-f_{k}^{z}} & \frac{c_{k}^{z}}{m g^{z}-f_{k}^{z}} & 0 & 0 \\
0 & 0 & -\frac{1}{m g^{z}-f_{k}^{z}} & \frac{c_{k}^{z}}{m g^{z}-f_{k}^{z}}
\end{array}\right],}
\end{gathered}
$$

$T$ being the sampling period. Note that the subscript ext was dropped from $\mathbf{f}_{k}$ which represents the external wrench at the $k$-th time interval. We can now formulate the walking pattern generator.

\section{MODEL PREDICTIVE CONTROL}

Model Predictive Control is a method of controlling a system such that future states are also taken into account. This makes it effective in producing walking motions, as demonstrated in [2], [3]. A common MPC methodology consists in iteratively applying the model over $N$ discrete steps, resulting in a new problem formulation where the previewed future states are a function of the current state and of the current and future control inputs. Doing so for (8) results in:

$$
\left\{\begin{array}{l}
\hat{\mathbf{C}}=\mathbf{U}_{x} \hat{\mathbf{c}}_{0}+\mathbf{U}_{u} \dddot{\mathbf{C}} \\
\hat{\mathbf{Z}}=\mathbf{O}_{x} \hat{\mathbf{c}}_{0}+\mathbf{O}_{u} \dddot{\mathbf{C}}+\mathbf{O}_{f} \hat{\mathbf{F}}
\end{array}\right.
$$

where the vectors $\hat{\mathbf{C}}, \hat{\mathbf{Z}}, \dddot{\mathbf{C}}, \hat{\mathbf{F}}$ are just a concatenation of states, outputs, controls and external forces respectively in the preview horizon. The matrices $\mathbf{U}_{x}, \mathbf{U}_{u}, \mathbf{O}_{x}, \mathbf{O}_{u}, \mathbf{O}_{f}$ can be obtained similarly to [3].

It will be useful to express the second equation of (9) in terms of the foot landing positions, to enable the controller to modify them as in [3]. This is done by expressing the global ZMP positions $\hat{\mathbf{Z}}$ through the ZMP positions $\mathbf{Z}$ in local coordinates of the supporting foot/feet such that:

$$
\hat{\mathbf{Z}}=\mathbf{V}_{\mathbf{p}_{0}}+\mathbf{V P}+\mathbf{R}_{\hat{\mathbf{p}}_{\mathbf{k}}} \mathbf{Z}
$$

where $\mathbf{P}$ is a vector of future foot landing positions expressed in a local frame assigned to the preceding foot positions. The expression $\mathbf{V}_{\mathbf{p}_{0}}+\mathbf{V P}$ produces positions of the feet in 
the global reference frame. Lastly, $\mathbf{R}_{\hat{\mathbf{p}}_{\mathbf{k}}}$ is a block diagonal matrix of the feet rotation matrices. This is also detailed in [3]. Note that the rotation matrices are separately predetermined/precomputed to preserve linearity [3].

For a walking pattern generator with automatic footstep placement, we can define the optimization argument $\mathbf{X}=$ $\left[\dddot{\mathbf{C}}^{\top} \mathbf{P}^{\top}\right]^{\top}$ and rewrite (9) and (10) as:

$$
\left\{\begin{array}{l}
\hat{\mathbf{C}}=\left[\begin{array}{ll}
\mathbf{U}_{u} & \mathbf{0}
\end{array}\right]\left[\begin{array}{l}
\dddot{\mathbf{C}} \\
\mathbf{P}
\end{array}\right]+\mathbf{U}_{x} \hat{\mathbf{c}}_{0} \\
\mathbf{Z}=\mathbf{R}_{\hat{\mathbf{p}}_{\mathbf{k}}}^{\top}\left[\begin{array}{ll}
\mathbf{O}_{u} & -\mathbf{V}
\end{array}\right]\left[\begin{array}{l}
\dddot{\mathbf{C}} \\
\mathbf{P}
\end{array}\right]+\mathbf{R}_{\hat{\mathbf{p}}_{\mathbf{k}}}^{\top}\left(\mathbf{O}_{x} \hat{\mathbf{c}}_{0}+\mathbf{O}_{f} \hat{\mathbf{F}}-\mathbf{V}_{\mathbf{p}_{0}}\right) .
\end{array}\right.
$$

More concisely, (11) can be written:

$$
\left\{\begin{array}{l}
\hat{\mathbf{C}}=\mathbf{S X}+\mathbf{s} \\
\mathbf{Z}=\mathbf{S}_{z} \mathbf{X}+\mathbf{s}_{z} .
\end{array}\right.
$$

With these, the objectives and constraints for optimization can be formulated. For now, we reconstruct a WPG similar to [3]. Firstly, the ZMP is constrained to be in the support polygon (with security margins) by defining the lower and upper bounds (denoted by the lower and upper bar):

$$
\underline{\mathbf{Z}} \leq \mathbf{Z} \leq \overline{\mathbf{Z}} .
$$

Using the second equation in (12) to expose the argument we have:

$$
\underline{\mathbf{Z}}-\mathbf{s}_{z} \leq \mathbf{S}_{z} \mathbf{X} \leq \overline{\mathbf{Z}}-\mathbf{s}_{z} .
$$

A control objective to track a reference CoM velocity is created via an appropriate selection matrix $\mathbf{I}_{v}$ such that:

$$
\dot{\mathbf{C}}=\mathbf{I}_{v} \hat{\mathbf{C}}=\mathbf{I}_{v}(\mathbf{S X}+\mathbf{s})=\mathbf{S}_{v} \mathbf{X}+\mathbf{s}_{v},
$$

as in [3]. The control objective can then be written as:

$$
\left\|\dot{\mathbf{C}}-\dot{\mathbf{C}}_{\mathrm{ref}}\right\|^{2}=\left\|\mathbf{S}_{v} \mathbf{X}+\mathbf{s}_{v}-\dot{\mathbf{C}}_{\mathrm{ref}}\right\|^{2}
$$

A second useful objective is to minimize the CoM jerk. Although not strictly necessary, this was shown to improve performance in [3]:

$$
\|\dddot{\mathbf{C}}\|^{2}=\left\|\left[\begin{array}{ll}
\mathbf{I} & 0
\end{array}\right] \mathbf{X}\right\|^{2}
$$

This is also in line with Linear-quadratic regulation, which places a cost on the control input to reduce it.

The third objective is to minimize the distance between the ZMP positions and the feet centers. Since the ZMP from (12) is defined with respect to the feet centers, this amounts to giving a vector of zeros as a reference such that the objective function is:

$$
\|\mathbf{Z}\|^{2}=\left\|\mathbf{S}_{z} \mathbf{X}+\mathbf{s}_{z}\right\|^{2}
$$

The idea behind this objective is that we prefer a change in the foot landing position over a change in the ZMP. This gives a better stability margin, since unknown disturbances could push the ZMP away from the target. Since a constraint of the control is to keep the ZMP within the support polygon, the most robust ZMP target location under unknown disturbances is in the middle of these bounds.
Finally, we need to add simple bounds for the foot positions such that:

$$
\underline{\mathbf{P}} \leq\left[\begin{array}{ll}
\mathbf{0} & \mathbf{I}
\end{array}\right] \mathbf{X} \leq \overline{\mathbf{P}}
$$

This constraint is optional when considering the walking pattern generator by itself. However, it plays an important role when considering the whole body control problem. In fact, it approximates the constraints of the whole body model into the WPG.

Finally, we can combine all the objectives and constraints into a single quadratic programming $(\mathrm{QP})$ problem. For clarity, the original variables are used below, although (14), (16), (17), (18), (19) express them as a function of the argument, making the construction of the standard QP straightforward. The standard WPG can then be described as:

$$
\begin{array}{ll}
\underset{\mathbf{X}}{\operatorname{argmin}} & \frac{\alpha}{2}\left\|\dot{\mathbf{C}}-\dot{\mathbf{C}}_{\text {ref }}\right\|^{2}+\frac{\beta}{2}\|\dddot{\mathbf{C}}\|^{2}+\frac{\gamma}{2}\|\mathbf{Z}\|^{2} \\
\text { subject to } & \underline{\mathbf{Z}} \leq \mathbf{Z} \leq \overline{\mathbf{Z}} \\
& \underline{\mathbf{P}} \leq \mathbf{P} \leq \overline{\mathbf{P}} .
\end{array}
$$

Note that weights are used to combine the objective functions. In cases when the objectives compete with each other, these weights slightly alter the expected performance. That is: a higher $\alpha$ allows better reference velocity tracking, a higher $\beta$ reduces CoM jerk and a higher $\gamma$ allows less ZMP movement.

From (20), it is not immediately apparent how the external wrench added in the model affects the final performance of the WPG. As mentioned before, (20) is based on [3]. Returning to (9), note that the external wrench only appears in the ZMP formulation. It then affects the QP in two areas: ZMP constraint, and ZMP centering objective. The first way to think about it is that the external wrench changes the ZMP value, as explained in [7]. However, taken another way, we can also say that the external wrench reshapes the ZMP bounds as :

$$
\begin{aligned}
\underline{\mathbf{Z}} & \leq \mathbf{Z}^{\prime}+\mathbf{Z}_{f} \leq \overline{\mathbf{Z}} \\
\underline{\mathbf{Z}}-\mathbf{Z}_{f} & \leq \mathbf{Z}^{\prime} \leq \overline{\mathbf{Z}}-\mathbf{Z}_{f}
\end{aligned}
$$

where $\mathbf{Z}_{f}$ represents the ZMP offset resulting from the external wrench and $\mathbf{Z}^{\prime}$ the classical ZMP. This is inline with a quadruped analysis when considering the task of a humanoid and human carrying an object together. The effect of the external wrench on the ZMP centering objective is to further change the foot landing position based on this ZMP change.

\section{SPECIFIC USE CASES IN PHYSICAL COLLABORATION}

From the base QP formulation (20), we can apply variations to better fit the expected type of physical interaction. In particular, a follower and a leader formulation are presented.

\section{A. Follower}

A follower acts based on the leader's intention. Previous works [5], [6] have indirectly used a simple damping control providing the reference velocity as $\dot{c}_{\text {ref }}=f / b$ and using the provided interface from (16). We can extend this to perform 
slightly more complex following behaviors by using a full mass-spring-damper model:

$$
f=m \ddot{c}+b \dot{c}+k c .
$$

Based on (22), an impedance control task in the $x, y$ plane can be defined directly in the WPG. Recalling that the state $\hat{\mathbf{C}}$ contains accelerations, velocities and positions, one simply needs to define an appropriate impedance parameter matrix $\mathbf{G}_{\text {mbk }}$. An appropriate selection matrix $\mathbf{S}_{x y}$ is also needed to select only the $f^{x}, f^{y}$ components such that:

$$
\left\|\mathbf{G}_{\mathrm{mbk}} \hat{\mathbf{C}}-\mathbf{S}_{x y} \hat{\mathbf{F}}\right\|^{2} \text {. }
$$

The argument can be obtained as in (16). For the follower configuration, this replaces the velocity objective and the external controller used to compute this reference in [5], [6]. The full QP can then be written as:

$$
\begin{array}{ll}
\underset{\mathbf{X}}{\operatorname{argmin}} & \frac{\alpha}{2}\left\|\mathbf{G}_{\mathrm{mbk}} \hat{\mathbf{C}}-\mathbf{S}_{x y} \hat{\mathbf{F}}\right\|^{2}+\frac{\beta}{2}\|\dddot{\mathbf{C}}\|^{2}+\frac{\gamma}{2}\|\mathbf{Z}\|^{2} \\
\text { subject to } & \underline{\mathbf{Z}} \leq \mathbf{Z} \leq \overline{\mathbf{Z}} \\
& \underline{\mathbf{P}} \leq \mathbf{P} \leq \overline{\mathbf{P}}
\end{array}
$$

Note that there are two important points about the external wrench term in the MPC. Firstly, recall in (9) that the external wrench terms appearing in the MPC are the future ones $\mathbf{f}_{1} \ldots \mathbf{f}_{N}$. In the cases where we use feedback from a force/torque sensor, what is obtained is the current external wrench $\mathbf{f}_{0}$. Therefore, we need to predict these future values. The prediction model leads to proactive behaviors in physical collaboration. The second important aspect is that the wrench is expressed in the CoM frame, whereas a sensor produces values in that sensor's frame. Here, we consider a quasi-static motion for doing this frame transformation.

\section{B. Leader}

For leading, a clear and independent intention is necessary. In [5], this was generated by another human operator providing the reference velocity commands needed in (16). If the full trajectory is known beforehand, a better objective for the leader may be trajectory rather than reference velocity tracking. This can be formulated as:

$$
\left\|g_{m}\left(\ddot{\mathbf{C}}_{\text {ref }}-\ddot{\mathbf{C}}\right)+g_{b}\left(\dot{\mathbf{C}}_{\text {ref }}-\dot{\mathbf{C}}\right)+g_{k}\left(\mathbf{C}_{\text {ref }}-\mathbf{C}\right)\right\|^{2} \text {. }
$$

To be concise, a suitable gain matrix can be used:

$$
\left\|\mathbf{G}_{\text {mbk }}\left(\hat{\mathbf{C}}_{\text {ref }}-\hat{\mathbf{C}}\right)\right\|^{2} \text {. }
$$

Another idea for leading is to place the external wrench as a part of the QP argument, such that $\mathbf{X}=\left[\dddot{\mathbf{C}}^{\top} \mathbf{P}^{\top} \hat{\mathbf{F}}^{\top}\right]^{\top}$. This changes (11) into:

$$
\left\{\begin{array}{l}
\hat{\mathbf{C}}=\left[\begin{array}{lll}
\mathbf{U}_{u} & \mathbf{0} & \mathbf{0}
\end{array}\right]\left[\begin{array}{c}
\dddot{\mathbf{C}} \\
\mathbf{P} \\
\hat{\mathbf{F}}
\end{array}\right]+\mathbf{U}_{x} \hat{\mathbf{c}}_{0} \\
\mathbf{Z}=\mathbf{R}_{\hat{\mathbf{p}}_{\mathbf{k}}}^{\top}\left[\begin{array}{lll}
\mathbf{O}_{u} & -\mathbf{V} & \mathbf{O}_{f}
\end{array}\right]\left[\begin{array}{c}
\dddot{\mathbf{C}} \\
\mathbf{P} \\
\hat{\mathbf{F}}
\end{array}\right]+\mathbf{R}_{\hat{\mathbf{p}}_{\mathbf{k}}}^{\top}\left(\mathbf{O}_{x} \hat{\mathbf{c}}_{0}-\mathbf{V}_{\mathbf{p}_{0}}\right) .
\end{array}\right.
$$

Note that only the $x$ and $y$ components of the wrench are in $\hat{\mathbf{F}}$ and that $\mathbf{O}_{f}$ contains $f_{\text {ext }}^{z}$, which will need to be predefined for the preview horizon.

As the force is part of the ZMP expression, this may allow the robot to balance itself by applying the appropriate forces. For safety, the applied wrench may need to be constrained:

$$
\underline{\hat{\mathbf{F}}} \leq \hat{\mathbf{F}} \leq \hat{\overline{\mathbf{F}}}
$$

and/or minimized as:

$$
\|\hat{\mathbf{F}}\|^{2}
$$

If both options are chosen, the full QP becomes:

$$
\begin{array}{ll}
\underset{\mathbf{X}}{\operatorname{argmin}} & \frac{\alpha}{2}\left\|\mathbf{G}_{\mathrm{mbk}}\left(\hat{\mathbf{C}}_{\text {ref }}-\hat{\mathbf{C}}\right)\right\|^{2}+\frac{\beta}{2}\|\dddot{\mathbf{C}}\|^{2} \\
& +\frac{\gamma}{2}\|\mathbf{Z}\|^{2}+\frac{\delta}{2}\|\hat{\mathbf{F}}\|^{2} \\
\text { subject to } & \underline{\mathbf{Z}} \leq \mathbf{Z} \leq \overline{\mathbf{Z}} \\
& \underline{\mathbf{P}} \leq \mathbf{P} \leq \overline{\mathbf{P}} \\
& \underline{\hat{\mathbf{F}}} \leq \hat{\mathbf{F}} \leq \hat{\overline{\mathbf{F}}} .
\end{array}
$$

Having the external wrench in the argument implies that this result needs to be tracked by the whole-body controller. To do this, we can use an impedance controller on the hands with force sensors. The same quasi-static assumption for the frame transformation is used.

\section{Simulations}

We tested the three WPG formulations in several simulations prior to the real robot experiments. Here, we show some of the more extreme cases which highlight the inclusion of the external wrench in the model and control strategy. The cases show motion/postural changes in the saggital plane, which is the usual walking direction for humans. It also has a larger stability margin due to the base of support (foot) being longer in that direction. This allows a larger allowable change of wrench in between WPG updates (100 ms).

We begin with the standard WPG in (20). Figure 1 clearly shows the effects of the external wrench. The leaning postures are not preprogrammed, but rather the result of tracking the CoM motion and footsteps generated by the WPG, which compensate the external wrench in each case. The external wrench is also added to the multi-body dynamics, to make the robot walk in this dynamics simulation.

The next simulation is that of the follower WPG in (24). Fig. 2 shows the plots of a critically damped system with stiffness of $50 \mathrm{~N} / \mathrm{m}$.

The last simulation is for the leader WPG in (30). In this test, we increase the weight $\beta$, penalizing the CoM motion. This implies that the robot will use the external wrench to balance itself, instead of swaying its CoM. Furthermore, forces and torques are constrained to be $\pm 20 \mathrm{~N}$ and $\mathrm{Nm}$ respectively. Lastly, the leader uses a B-spline to build its trajectory of $1 \mathrm{~m}$ forward in 10 seconds. Figure 3 shows that the CoM oscillation in the $y$ axis of other WPGs is almost gone. In place of this, the generated reference external wrench needs to be applied. We anticipate that in 


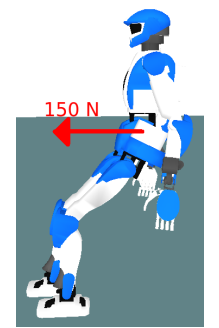

(a) $150 \mathrm{~N}$ of force (forwards) applied on the robot CoM while walking with $-0.1 \mathrm{~m} / \mathrm{s}$ reference velocity (backwards)

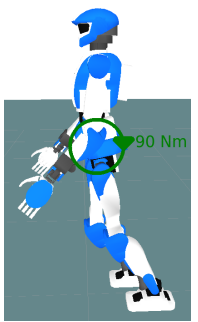

(b) $-90 \mathrm{Nm}$ of torque (clockwise) applied on the robot CoM while walking with $0.1 \mathrm{~m} / \mathrm{s}$ reference velocity (forwards)

Fig. 1. Simulations of the standard WPG

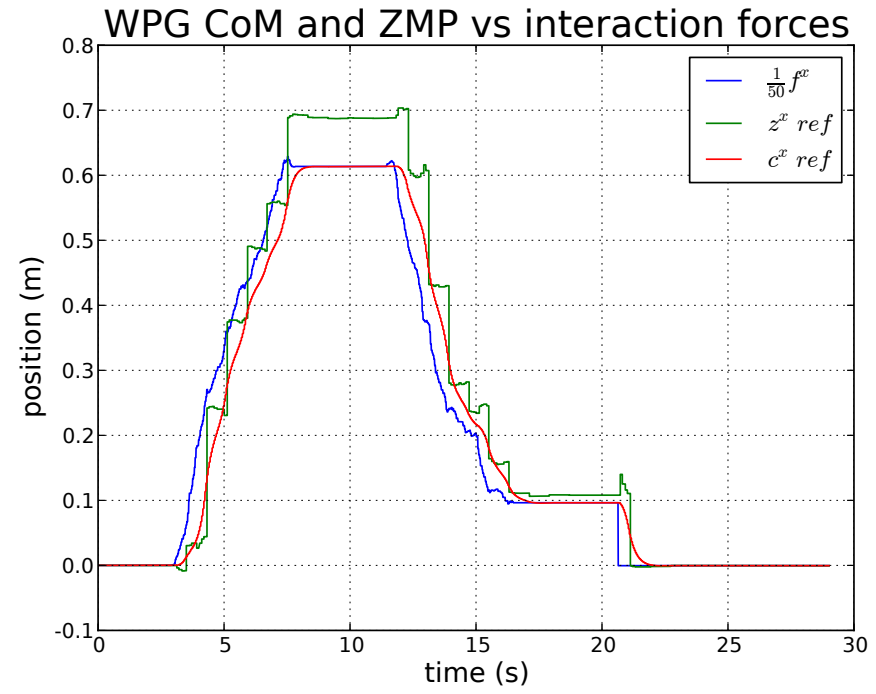

Fig. 2. Simulation data for critically damped follower (stiffness $=50 \mathrm{~N} / \mathrm{m}$ ).

future works this property will be very useful in the case of collaborative carrying, as the CoM sway of the robot can disturb the human partner.

\section{REAL ROBOT EXPERIMENT AND RESULTS}

After verification on simulations, we also test the individual cases on the real robot. Details about running a walking pattern generator on the HRP-4 can be found in [16]. In this section, we propose a switching scenario, similar to [5], which shows the two new WPG formulations, (24) and (30), on the real robot. The robot starts out as a follower and due to some condition, it switches to being a leader. Some plausible switching conditions are:

- interaction force limits are exceeded

- workspace limits are exceeded

- losing balance (e.g., through capturability limits [17]).

In [5], the switch was made at a predetermined time. Here, a maximum interaction force is the switching condition, although several conditions can be used together. Figure 4 shows a photo during this test, while Fig. 5 shows the generated references of the WPG, along with the interaction forces. The switching condition is exceeding $25 \mathrm{~N}$, which happens around the 50 second mark. After this, the leader
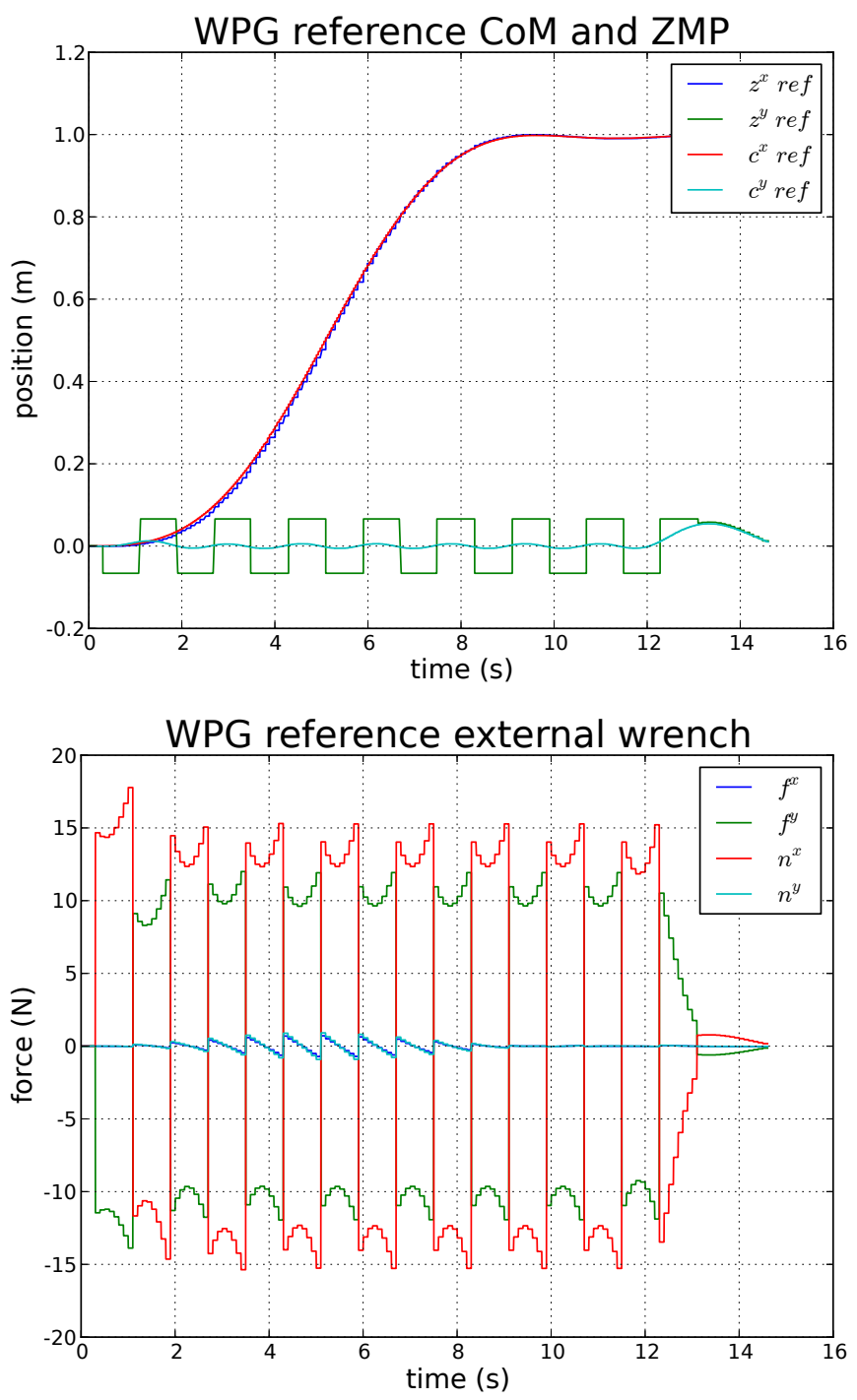

Fig. 3. Simulation data for a leader with trajectory of $1 \mathrm{~m}$ forward in $10 \mathrm{~s}$.

trajectory of going back $0.2 \mathrm{~m}$ in $10 \mathrm{~s}$ is executed. Note that in this experiment, the torques were constrained to be $0 \mathrm{Nm}$, while forces are allowed to be in the range $\pm 20 \mathrm{~N}$. This simplifies the force control problem for one hand.

For this test, the follower case was done with damping only for simplicity. The results show that even with the sensor noise, the robot can follow the intent of the human leader. On the leader case, note that the generated reference force is quite low (the gains were not tuned as extremely as in the simulation example). This allows the robot to balance by itself (not relying on the force control). This choice arose from the difficulty in implementing a high-fidelity force control on a position controlled robot.

\section{CONCLUSION AND Future WORK}

In this paper, walking pattern generators are designed for use in physical interaction and collaboration. To this end, the dynamic walk model is revisited, by adding an external wrench term. From this model, we formulate a controller 

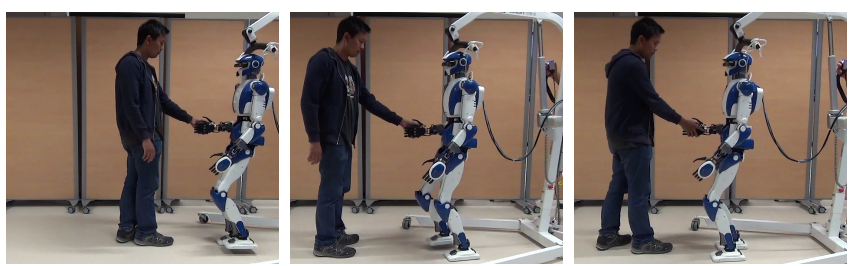

Fig. 4. Screenshots from the video of the real-robot experiment

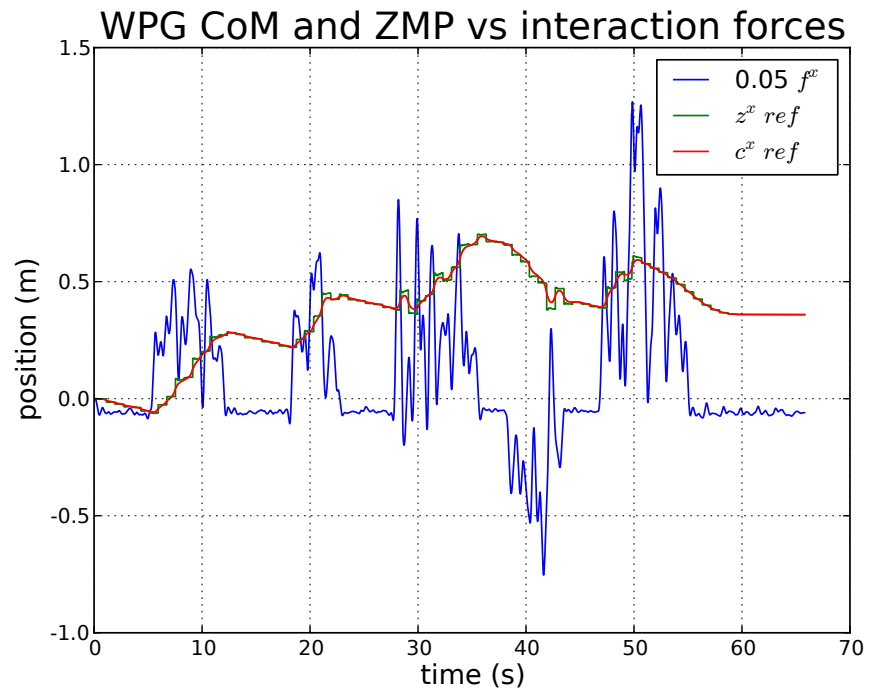

Fig. 5. Data from the switching experiment

that is consistent with the desired physical interaction behavior. This means that several different formulations can be designed, using a mix and match approach of the objective functions and constraints in the optimization problem. We then show results both in simulation and a real experiment. This work is part of our larger goal to enable humanoids to collaboratively carry objects with humans [18].

Although our approach is shown to work well, several issues are still present, along with different paths for further improvement. One key issue we saw from real experiments is the need for a high-fidelity force control in the leader WPG. A key improvement for the follower WPG that we only briefly mentioned is the wrench prediction model for better proactive behaviors. A limitation of this work is that the wrench is simply predicted to be constant throughout the preview horizon. Furthermore, the WPG presented here was from a known simplified form, but the core concepts presented here are not conflicting with improvements such as [19] which added robustness, allowing stair climbing. Apart from improving the WPG itself, its integration to whole-body control can also be improved with works such as [20]. The weight tuning issue also needs to be considered depending on the particular problem.

\section{ACKNOWLEDGEMENT}

This work is supported in part by the FP7 RoboHow.Cog project (www.robohow.eu) and the PSPC ROMEO 2 (www.projetromeo.com) national project.

\section{REFERENCES}

[1] M. Vukobratović and B. Borovac, "Zero-Moment Point - Thirty Five Years Of Its Life," International Journal of Humanoid Robotics, vol. 1, no. 1, pp. 157-173, 2004.

[2] S. Kajita, F. Kanehiro, K. Kaneko, K. Fujiwara, K. Harada, K. Yokoi, and $\mathrm{H}$. Hirukawa, "Biped walking pattern generation by using preview control of zero-moment point," in IEEE International Conference on Robotics and Automation, vol. 2, pp. 1620-1626, 2003.

[3] A. Herdt, H. Diedam, P.-B. Wieber, D. Dimitrov, K. Mombaur, and M. Diehl, "Online walking motion generation with automatic footstep placement," Advanced Robotics, vol. 24, no. 5-6, pp. 719-737, 2010

[4] K. Yokoyama, H. Handa, T. Isozumi, Y. Fukase, K. Kaneko, F. Kanehiro, Y. Kawai, F. Tomita, and H. Hirukawa, "Cooperative works by a human and a humanoid robot," in IEEE International Conference on Robotics and Automation, vol. 3, pp. 2985-2991, 2003.

[5] A. Bussy, P. Gergondet, A. Kheddar, F. Keith, and A. Crosnier, "Proactive behavior of a humanoid robot in a haptic transportation task with a human partner," in IEEE International Symposium on Robot and Human Interactive Communication, pp. 962-967, 2012.

[6] D. J. Agravante, A. Cherubini, A. Bussy, P. Gergondet, and A. Kheddar, "Collaborative Human-Humanoid Carrying Using Vision and Haptic Sensing," in IEEE International Conference on Robotics and Automation, pp. 607-612, May 2014.

[7] K. Nishiwaki and S. Kagami, "Online walking control system for humanoids with short cycle pattern generation," International Journal of Robotics Research, vol. 28, no. 6, pp. 729-742, 2009.

[8] K. Harada, S. Kajita, K. Kaneko, and H. Hirukawa, "Dynamics and balance of a humanoid robot during manipulation tasks," IEEE Transactions on Robotics, vol. 22, pp. 568-575, June 2006.

[9] H. Hirukawa, S. Hattori, K. Harada, S. Kajita, K. Kaneko, F. Kanehiro, K. Fujiwara, and M. Morisawa, "A universal stability criterion of the foot contact of legged robots - adios ZMP," in IEEE International Conference on Robotics and Automation, pp. 1976-1983, May 2006.

[10] S. Nozawa, I. Kumagai, Y. Kakiuchi, K. Okada, and M. Inaba, "Humanoid full-body controller adapting constraints in structured objects through updating task-level reference force," in IEEE/RSJ International Conference on Robots and Intelligent Systems, pp. 34173424 , Oct 2012.

[11] T. Takubo, K. Inoue, and T. Arai, "Pushing an Object Considering the Hand Reflect Forces by Humanoid Robot in Dynamic Walking," in IEEE International Conference on Robotics and Automation, pp. 1706-1711, April 2005.

[12] A. Ibanez, P. Bidaud, and V. Padois, "Unified preview control for humanoid postural stability and upper-limb interaction adaptation," in IEEE/RSJ International Conference on Robots and Intelligent Systems, pp. 1801-1808, Oct 2012.

[13] C. Ott, B. Henze, and D. Lee, "Kinesthetic teaching of humanoid motion based on whole-body compliance control with interactionaware balancing," in IEEE/RSJ International Conference on Robots and Intelligent Systems, pp. 4615-4621, Nov 2013

[14] M. Murooka, S. Nozawa, Y. Kakiuchi, K. Okada, and M. Inaba, "Whole-body pushing manipulation with contact posture planning of large and heavy object for humanoid robot," in IEEE International Conference on Robotics and Automation, pp. 5682-5689, May 2015.

[15] P.-B. Wieber, R. Tedrake, and S. Kuindersma, "Modeling and control of legged robots," in Springer handbook of robotics (B. Siciliano and O. Khatib, eds.), ch. 48, Springer, second ed., (to appear).

[16] S. Kajita, M. Morisawa, K. Miura, S. Nakaoka, K. Harada, K. Kaneko, F. Kanehiro, and K. Yokoi, "Biped walking stabilization based on linear inverted pendulum tracking," in IEEE/RSJ International Conference on Robots and Intelligent Systems, pp. 4489-4496, Oct 2010

[17] T. Koolen, T. de Boer, J. Rebula, A. Goswami, and J. Pratt, "Capturability-based analysis and control of legged locomotion, Part 1: Theory and application to three simple gait models," International Journal of Robotics Research, vol. 31, no. 9, pp. 1094-1113, 2012.

[18] D. J. Agravante, A. Cherubini, A. Sherikov, P.-B. Wieber, and A. Kheddar, "Human-Humanoid Collaborative Carrying," International Journal of Robotics Research, (submitted to).

[19] C. Brasseur, A. Sherikov, C. Collette, D. Dimitrov, and P.-B. Wieber, "A robust linear MPC approach to online generation of 3D biped walking motion," in IEEE-RAS International Conference on Humanoid Robots, pp. 595-601, Nov 2015.

[20] A. Sherikov, D. Dimitrov, and P.-B. Wieber, "Whole body motion controller with long-term balance constraints," in IEEE-RAS International Conference on Humanoid Robots, pp. 444-450, Nov 2014. 